\title{
Dynamics of Hydrogen Molecules Produced on a Graphite Surface
}

\author{
Yoonhee Ko, Jongbaik Ree, ${ }^{\dagger}$ Yoo Hang Kim, ${ }^{\dagger}$ and Hyung Kỵu Shin ${ }^{\ddagger}$ \\ Department of Chemistrv Education, Chonnam National Lniversin. Gwangin 500-757, Korea \\ ${ }^{\dagger}$ Department of Chemistry and Center for Chemical Dunamics, Ihha University. Inchon $402-751$, Korea \\ ${ }^{\ddagger}$ Deparment of Chemistry, Chiversitv of Nevada. Reno. Vevada 89557 , USt \\ Received Mav 29, 2002
}

\begin{abstract}
We have studied the dynamics of energy-rich hydrogen molecules produced on a graphite surface through $\mathrm{H}(\mathrm{g})$ $+\mathrm{H}(\mathrm{ad}) / \mathrm{C}(\mathrm{gr}) \rightarrow \mathrm{H}_{\beth}(\mathrm{g})+\mathrm{C}(\mathrm{gr})$ at thermal conditions mimicking the interstellar medium using a classical trajectory procedure. The recombination reaction of gaseous $\mathrm{H}$ atom at $100 \mathrm{~K}$ and the adsorbed $\mathrm{H}$ atom on the interstellar graphite grains at $10 \mathrm{~K}$ efficiently takes place on a subpicosecond time scale with most of the reaction exothermicity depositing in the product vibration, which leads to a strong vibrational population inversion. The molecules produced in nearly end-on geometry where $\mathrm{H}(\mathrm{g})$ is positioned below $\mathrm{H}(\mathrm{ad}) \mathrm{rotate}$ clockwise and are more highly rotationally excited. but in low-lying vibrational levels. The rotational axis of most of the molecule rotating clockwise is tilted from the surface normal by more than $30^{\circ}$, the intensity peaking at $35^{\circ}$. The molecules produced when $\mathrm{H}(\mathrm{ad})$ is close to the surface rotate counter-clockwise and are weakly rotationally excited. but highly vibrationally excited. These molecules tend to align their rotational aves parallel to the surface. The number of molecules rotating clockwise is eight times larger than that rotating counter-clockwise.
\end{abstract}

Key Words : Graphite, Hydrogen, Classical trajectory. Interstellar. Vibration

\section{Introduction}

The recombination reaction of gas-phase atonic hydrogen with adsorbed hydrogen atoms on a solid surface has been known to occur in a direct gas-adatom collision. ${ }^{1.7}$ An amorphous graphite surface is one such system on which hydrogen recombination has been studied extensively in recent years as a result of astrophysical interests. Atomic hydrogen is by far the most abundant gaseous constituent in the interstellar medium. When graphite grains are highly porous, hydrogen atonss which interact weakly with the surface sites can be trapped interstitially. In such an enviromment. the weakly adsorbed atom can be formed on a graphite site ${ }^{\S}$ where it interacts with the incident gas atom. and their recombination on interstellar dust grains is believed to be the leading hypothesis for the formation of $\mathrm{H}_{2}$ in cold interstellar clouds. ${ }^{\text {-1 }} 15$

In ref. 16. we have studied the reaction of gas-phase atomic hydrogen reacting with adatoms in the limits of chemisorption and physisorption. In the chemisorption limit the adatom-surface interaction is considered to be the typical $\mathrm{C}-\mathrm{H}$ bond energy $(3.30 \mathrm{eV})$, whereas in the physisorption limit it is a weak van der Waals (vdW) interaction (39.3 $\mathrm{meV}$ ). On the other hand. density functional theory (DFT) shows ${ }^{17}$ that hydrogen atoms exclusively chemisorb on top of a carbon at short range (around $1.5 \AA$ ). the region which has been considered in recent studies. ${ }^{18-21}$ The DFT study also shows the existence of a physisorption region around $3 \mathrm{~A}$ and the presence of both adsorption regions separated by a barrier. ${ }^{17}$ The latter study indicates that the physisorption

\footnotetext{
*Author to whom correspondence should be addressed. E-mail: jbreetchonnam.ackr
}

description is consistent with the vdW model. We have already explored the role of this region in the formation of hydrogen molecules. ${ }^{16}$

In this paper. as an extension of the work in ref. 16, we study the recombination dynamics of hydrogen atoms on a graphite surface at the thenmal conditions appropriate to the interstellar medium. where the interstellar grains are near 10 $\mathrm{K}$ and the gas is at $10-100 \mathrm{~K}^{18}$ using the physisorption region consistent with the $\mathrm{vdW}$ model. ${ }^{16}$ In the physisorption limit, vibrational and rotational states are found to be highly excited. The major focus of our study is to elucidate the vibrational and rotational distributions and extent to which these distributions are related to rotational orientation and alignment in the interstellar medium. From the solution of equations of motion of primary syistem atoms. we first establish the occurrence of reaction and extents of vibrational and rotational excitation. We then study the dependence of reaction probability on the impact parameter. rotational and vibrational population distributions in relation to the orientation of product molecules.

\section{Interaction Model}

The incident gas atom $\mathrm{H}(\mathrm{g})$ approaches a graphite surface on which hydrogen atoms are adsorbed. We consider the reaction between $\mathrm{H}(\mathrm{g})$ and $\mathrm{H}(\mathrm{ad})$ preadsorbed on the center atom $C_{[i j)}$. which is surrounded by 12 nearby carbon atoms of three benzene rings. $\mathrm{H}(\mathrm{g})$ is in interaction with all 12 surface sites $\mathrm{C}_{\mathrm{ij},}, i=1-12$. Figure 1 shows the numbering of these atoms on the surface layer and definitions of the pertinent coordinates. The reaction zone atoms are the incident gas atom $\mathrm{H}(\mathrm{g})$. the preadsorbed atom $\mathrm{H}(\mathrm{ad})$ and the center 


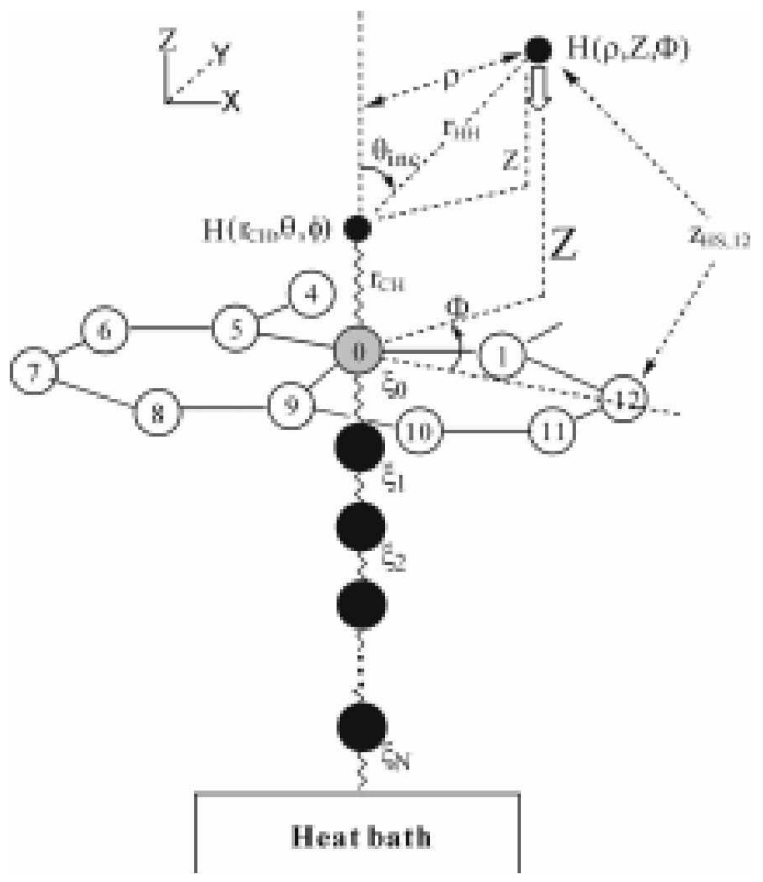

Figure 1. Interaction model showing the gas-phase atom $\mathrm{H}(\mathrm{g})$ denoted by $\mathrm{H}(\rho, Z, \Phi)$, interacting with the adsorbed atom $\mathrm{H}\left(r_{\mathrm{cH}}, \theta, \phi\right), \mathrm{H}(\mathrm{ad})$, adsorbed on $\mathrm{C}_{10) !}$, which is coupled to the $k$ atom chain and is surrounded by 12 sites. The Nth atom of the chain is coupled to the heat bath. The Hig)-to- $\mathrm{C}_{\text {rii }}$ distance is denoted by $z_{\mathrm{HS} \text {. }}$. For clarity, the 2 nd and 3 rd surface atoms are not showit.

graphite atom $\mathrm{C}_{(0)}$. The model considers $\mathrm{C}_{(0)}$ as the Oth member of the $(N+1)$-atom chain which links the reaction zone to the heat bath. providing a quasiphysical picture of energy flow between the reaction zone and the chain atoms and. in turn. between the chain and the heat bath. 22.23

A total of six degrees of freedom is necessary to describe the motions of $\mathrm{H}(\mathrm{g})$ and $\mathrm{H}(\mathrm{ad})$ above the surface. Although it is straightforward to transform these coordinates to the center-of-mass and relative coordinate sy'stems. we find that it is convenient to describe the collision system in terms of the atomic coordinates for the gas atom $\mathrm{H}\left(x_{\mathrm{H}}, y_{\mathrm{H}} z_{\mathrm{H}}\right)$ and for the adatom $\mathrm{H}\left(x_{\mathrm{H}}, y_{\mathrm{H}} \cdot z_{\mathrm{H}}\right){ }^{16.23}$ The $\mathrm{H}(\mathrm{ad})$ coordinates are $x_{\mathrm{H}}$. $=r_{\mathrm{CH}} \sin \theta \cos \phi . y_{\mathrm{H}}=r_{\mathrm{CH}} \sin \theta \sin \phi$. and $z_{\mathrm{H}}=r_{\mathrm{H}} \cos \theta$, i.e.

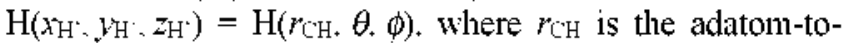
$\mathrm{C}_{(i)}$ distance and $\theta, \phi$ determine the orientation of the adatom- $\mathrm{C}_{(i)}$ bond with respect to the surface normal and the $x$-axis. respectively: see Figure 1 . The $\mathrm{H}(\mathrm{g})$-to-H(ad) distance $r_{\mathrm{HH}}$ is $\left(\rho^{2}-z^{2}\right)^{1:}$. where $\rho$ is the distance between $H(\mathrm{~g})$ and $H(\mathrm{ad})$-surface normal axis. and $z$ is the vertical distance from $\mathrm{H}(\mathrm{g})$ to the horizontal line determining the position of $\mathrm{H}(\mathrm{ad})$. Note that the initial $(\mathrm{t} \rightarrow-\infty)$ value of $\rho$ is the impact parameter $b$. The projection of $r_{\mathrm{HH}}$ on the surface plane is oriented by the angle $\Phi$ from the $x$ axis. Thus. the coordinate $\left(x_{\mathrm{H}}, y_{\mathrm{H}}, z_{\mathrm{H}}\right)$ can be transformed into the cylindrical system $(\rho, Z$. $\Phi)$. where $Z$ is the normal $\mathrm{H}(\mathrm{g})$-tosurface distance. The vertical distance $z$ is then $z=Z$ $r_{C H} \cos \theta$. The initial state of the reaction zone is the gas atom approaching the surface at the initial condition of $(\rho, Z, \Phi)$ with the collision energy $E$ when the adatom takes the initial values of $\left(r_{\mathrm{CH},}, \theta, \phi\right)$. Thus. the occurrence of each event can be determined by studying the time evolution of the $\mathrm{H}(\mathrm{g})$ surface distance $Z$, the $\mathrm{H}(\mathrm{ad})$-surface bond distance $r_{\mathrm{CH}}$ and the $\mathrm{H}(\mathrm{g})$-to-H(ad) distance $r_{\mathrm{HH}}$ for the ensemble of gas atoms approaching the surface from all directions.

Each reactive events can be described in terms of the interactions of $\mathrm{H}(\mathrm{g})$ with $\mathrm{H}(\mathrm{ad}) . \mathrm{C}_{(0)}$. 12 adjacent carbon atoms and $N$ chain atoms. We construct an analytical form of the potential energy surface (PES) by combining a modified London-Eyring-Polanyi-Sato (LEPS) potential energy function with the $\theta, \dot{\phi}$-hindered rotational motions and the harmonic motions of the $(\mathrm{N}+\mathrm{l})$-chain atoms including the vibration of $\mathrm{C}_{(i 0)}{ }^{23}$ The complete PES is

$$
\begin{aligned}
& U=\left\{Q_{\mathrm{HH}}-Q_{\mathrm{HC}}+Q_{\mathrm{HS}}-\left[A_{\mathrm{HH}^{2}}{ }^{2}-A_{\mathrm{HC}}{ }^{2}+A_{\mathrm{HS}}{ }^{2}-A_{\mathrm{HH}} A_{\mathrm{HC}}\right.\right. \\
& \left.\left.-\left(A_{\mathrm{HH}} A_{\mathrm{HC}}\right) A_{\mathrm{HS}}\right]^{1: 2}\right\}+1 / 2 k_{\theta}\left(\theta-\theta_{\mathrm{e}}\right)^{2}+1 / 2 k_{\theta}\left(\phi-\phi_{\mathrm{e}}\right)^{2} \\
& +\sum\left(1 / 2 \mathrm{M}_{C}\left(\omega_{\mathrm{e}}^{2} \xi_{l}^{2}\right)\right. \\
& +\sum_{3}^{3} \text { (terms of type } 1 / 2 \mathrm{M}_{C} \omega_{\mathrm{k}, 1}{ }^{2} \xi_{1-1} \xi_{\text {). }} \\
& 1 / 2 \mathrm{M}_{C}\left(\mathrm{ot}_{\mathrm{t}+1}{ }^{2} \xi_{1} \xi_{\mathrm{j}}-1\right. \text {. etc.). }
\end{aligned}
$$

where $k_{\theta}$ and $k_{\phi}$ are force constants. $\theta_{\mathrm{e}}$ and $\phi_{e}$ are the equilibrium angles. $\mathrm{M}_{\odot}$ is the mass of the carbon atom. $\xi$ 's are the vibrational coordinates of the $(N+1)$ atoms. $\omega_{\mathrm{e}}$ is the Einstein frequency. and $\omega_{\mathrm{k}}$ is the coupling frequency characterizing the chain. The explicit forms of the coulomb terms $(Q$ 's) and exchange tenms $(A \mathrm{~s})$ written in the Morsetype functions are well known. These energy terms contain the adjustable Sato parameters ( $\Delta$ 's). In the reaction zone. we have three interactions. namely $\mathrm{H}(\mathrm{g})-\mathrm{H}(\mathrm{ad}) . \mathrm{H}(\mathrm{ad})-\mathrm{C}_{(0)}$ and $\mathrm{H}(\mathrm{g})-\mathrm{C}_{(\mathrm{o})}$. For these interactions. the coulomb and exchange energies are

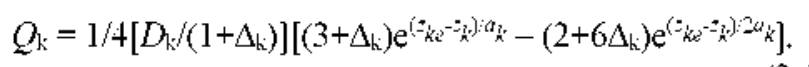

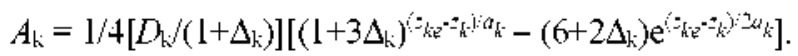

where $k=\mathrm{HH}$. $\mathrm{H}(\mathrm{ad}) \mathrm{C}_{(0 \mathrm{ij})}$ and $\mathrm{H}(\mathrm{g}) \mathrm{C}_{(0 \mathrm{i})}$ for $\mathrm{H}(\mathrm{g})-\mathrm{H}(\mathrm{ad})$. $\mathrm{H}(\mathrm{ad})-\mathrm{C}_{\mathrm{C}}$ and $\mathrm{H}(\mathrm{g})-\mathrm{C}_{(0)}$, respectively. Here, $\mathrm{z}_{\mathrm{ke}}$ is the equilibrium value of the $k$ th atom-atom distance $z_{\mathrm{k}}$ and $a_{\mathrm{k}}$ is the Morse-type range parameter. The $\mathrm{H}(\mathrm{g})-\mathrm{H}(\mathrm{ad}) . \mathrm{H}(\mathrm{ad})-\mathrm{C}_{(0 i)}$ and $\mathrm{H}(\mathrm{g})-\mathrm{C}_{(0)}$ distances are

$$
\begin{aligned}
& z_{\mathrm{HH}}=\left\{\rho^{2}+\left[Z-\left(d_{\mathrm{CH}}+x_{\mathrm{CH}}\right) \cos \theta\right]^{2}\right\}^{1: 2} . \\
& z_{\mathrm{HC}}=\left(d_{\mathrm{CH}}+x_{\mathrm{CH}}\right)+\xi_{\mathrm{O}} \text {. } \\
& z_{\mathrm{HS}(\mathrm{i})}=\left\{Z^{2}+\left[\rho \sin \phi+\left(d_{\mathrm{CH}}+x_{\mathrm{CH}}\right) \sin \phi\right]^{2}\right. \\
& \left.+\left[\left(d_{C \mathrm{H}}+x_{C \mathrm{H}}\right) \sin \theta+\cos \Phi\right]^{\hat{2}}\right\}^{1: 2},
\end{aligned}
$$

respectively: where $x_{\mathrm{CH}}$ is the displacement of the $\mathrm{H}(\mathrm{ad})-\mathrm{C}_{(0 i)}$ distance from its equilibrium value $d_{\mathrm{CH}}$ i.e.. $r_{\mathrm{CH}}=d_{\mathrm{CH}}+x_{\mathrm{CH}}$. We now add the 12 terms of the interaction between $\mathrm{H}(\mathrm{g})$ and adjacent surface sites to $Q_{\mathrm{HS}(\mathrm{i})}$ and $A_{\mathrm{HS}(0)}$ and refer to the sum as the $\mathrm{H}(\mathrm{g})$-surface interaction: 


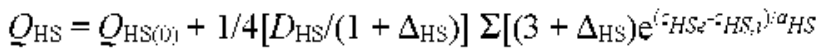

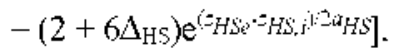

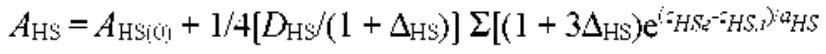

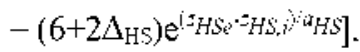

where $z_{\mathrm{HS} . \mathrm{i}}$ is the distance between $\mathrm{H}(\mathrm{g})$ and the $i$ th carbon atom. Since each $\mathrm{H}(\mathrm{g})$-to-surface atom distance $z_{\mathrm{HS}, \mathrm{i}}$ is $z_{\mathrm{HS} .}\left(r_{C \mathrm{H}}, \theta, \phi, \rho, Z, \Phi\right)$, the potential energy surface has the functional dependence $U\left(r_{\mathrm{CH}}, \theta, \phi . \rho . Z . \Phi,\{\xi\}\right)$, where $\{\xi\}=$ $\left(\xi_{1}, \xi_{1}, \cdots, \xi_{0}\right)$ for the vibrational coordinates of the $(N+1)$ chain atoms.

For the $\mathrm{H}-\mathrm{H}$ interaction, the binding energy is $D_{0}^{\mathrm{C}}=4.434$ $\mathrm{eV}$ at $z_{\mathrm{HHe}}=0.7414 \AA^{2-4}$ With the frequency $a_{\mathrm{HH}} / 2 \pi \mathrm{c}=4401$ $\mathrm{cm}^{-1}$. 24 we find the exponential range parameter $a_{\mathrm{HH}}=$ $\left(D_{\mathrm{HH}} / 2 \mu_{\mathrm{HH}}\right)^{1: 0 / \omega_{\mathrm{HH}}}=0.257 \AA$. where $D_{\mathrm{HH}}=D_{\mathrm{i}}^{\mathrm{i}}+\mathrm{l} / 2 \hbar \omega_{\mathrm{HH}}$ and $\mu_{\mathrm{HH}}$ is the reduced mass

The vibrational frequency of the van der Waals bond between $H(\mathrm{ad})$ and the graphite surface can be determined using the inverse-power-law potential $V^{\prime}\left(r_{\mathrm{CH}}\right)=D_{\text {vdv }}$ $\times\left[\left(\sigma / r_{\mathrm{CH}}\right)^{110}-\left(\sigma / r_{\mathrm{CH}}\right)^{6}\right]$ in the WKB eigenvalue expression ${ }^{35}$

$$
\frac{(8 \mu)^{1: 2}}{h} \int_{r_{C H}^{*}}^{r_{C H}^{*}}\left[E_{r}-D_{H S}-V\left(r_{C H}\right)\right]^{1: 2} d r_{C H}=\left(v+\frac{1}{2}\right) .
$$

where $r_{\mathrm{CH}}^{*}$ and $r_{\mathrm{CH}}^{* *}$ are the outer and inner turning points. respectively. Using $\sigma=3.38 \AA$ and the well depth $D_{\text {vdw }}=$ $39.3 \mathrm{meV}^{8}$ we find the vibrational frequency $188 \mathrm{~cm}^{-1}$ for the weak $\mathrm{H}(\mathrm{ad})-\mathrm{C}_{i(\mathrm{i} i}$ bond. The range parameter for the $\mathrm{vdW}$ bond is found to be $0.387 \AA$. We use the same Sato parameters as in Ref. 16. The set is $\Delta_{\mathrm{HH}}=0.30$ for $\mathrm{H}(\mathrm{g})$ -

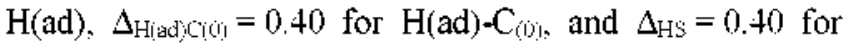
$\mathrm{H}(\mathrm{g})$ to each of the 13 surface-layer graphite atoms.

To study the reactive event, we follow the time evolution of the reaction system by integrating the equations of motion. which describe the motions of the reaction-zone atoms and $N$-chain atoms. An intuitive way to treat the dynamics of the reaction involving many surface atons is to solve the motions of the reaction-zone and $N$-chain atoms in the presence of $12 \mathrm{H}(\mathrm{g})-\mathrm{C}$ interactions governed by the molecular time scale generalized Langevin set of the equations of motion. $=2$ The details of the formulation of these equations have been described elsewhere ${ }^{2}$ but will be reviewed briefly here with several relevant expressions. The equations of motion for the gas atom and adatom are in the form

$$
m_{j} \ddot{Y}_{j}(t)=-\partial U\left(r_{C H} \cdot \theta \cdot \phi \cdot \rho, Z \cdot \Phi \cdot\{\xi\}\right) / Y_{j} .
$$

where $Y_{j}$ s are $Z, p . \Phi r_{\mathrm{CH}}, \theta \phi$ for $j=1.2, \ldots .6$. respectively. with $m_{1}=m_{\mathrm{H}}, m_{2}=\mu_{\mathrm{HH}}, m_{3}=\dot{I}_{\mathrm{HH}} . m_{4}=I_{\mathrm{HH}}$. and $m_{5}=m_{6}$ $=I_{\mathrm{HH}}$. Here $\mu$ is the reduced mass and $I$ is the moment of inertia. For the $(\mathrm{N}+1)$-atom chain dynamics. we have ${ }^{33}$

$$
\begin{aligned}
\ddot{\xi}_{(i}(t)= & -\omega_{e j}^{2} \xi_{(i)}(t)+\omega_{c 1}^{2} \xi_{1}(t) \\
& -M_{c}^{1} \partial U\left(z_{H S_{3}}, \theta . \phi . \rho . Z . \Phi .\{\xi\}\right) / \partial \xi_{0}
\end{aligned}
$$

$$
\begin{gathered}
\ddot{\xi}_{j}(t)=-\omega_{e j}^{2} \xi_{j}(t)+\omega_{c j}^{2} \xi_{j-1}(t)+\omega_{c j-1}^{2} \xi_{j+1}(t) . \\
j=1.2 \ldots N-1 \\
\xi_{N(t)}=-\Omega_{N}^{2} \xi_{N}(t)+\omega_{c N}^{2} \xi_{N-1}(t)-\beta_{N+1} \xi_{N}(t)+f_{N+1}(t)
\end{gathered}
$$

Here, $\Omega_{N}$ is the adiabatic frequency and $f_{k+1}(\mathrm{t})$ determines the random force on the primary system arising from thermal fluctuation in the heat bath. The friction coefficient $\beta_{N-1}$ is very close to $\pi \omega_{2} / 6$. where $\omega_{2}$ is the Debye frequency. The Debye temperature of graphite is $420 \mathrm{~K}^{27}$ All values of the frequencies and friction coefficients have been presented elsewhere. ${ }^{28}$ The initial conditions required to solve these equations have already been given in an earlier paper ${ }^{2 i}$

The numerical procedures include extensive use of Monte Carlo routines to generate random numbers for initial conditions. The first step is to sample collision energies $E$ from a Maxwell distribution at the gas temperature $T_{\mathrm{g}}$ and to weight the initial energy of $\mathrm{H}(\mathrm{ad})$-surface and all chain atom vibrations by a Boltzmann distribution at the surface temperature $T_{s}$. In sampling impact parameters. we take the flat range of $0<b<b_{\text {max }}$, where $b_{\text {max }}=2 d_{C C}=2.842 \AA$. Also sampled are the initial values $\theta_{1}$. $\phi_{!}$and $\Phi_{10}$. Thus. each

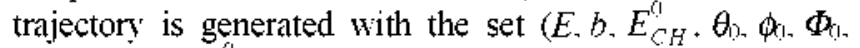
$\left\{\xi \xi_{0}\right)$. where $E_{C H}^{(!)}$is the initial energy of the adatom-surface vibration and $\left\{\xi_{c \mid}\right.$ represents the initial values of $\left\{\xi_{0 .}, \xi_{1} \ldots .\right.$. $\xi_{\mathrm{N}}$. The total number of trajectories sampled is 30000 . We follow each trajectory for $50 \mathrm{ps}$. which is a sufficiently long time for $\mathrm{H}_{2}(\mathrm{~g})$ to recede from the influence of surface interaction. to confirm the occurrence of a reactive event forming $\mathrm{H}_{2}(\mathrm{~g})$. Furthermore. we confirm that each trajectory can be successfully back-integrated in the computational procedure. We take the chain length of $N=10^{28}$

\section{Results and Discussion}

A. Reaction Probability. The probability of $\mathrm{H}_{2}$ formation in the reaction $\mathrm{H}(\mathrm{g})+\mathrm{H}(\mathrm{ad}) / \mathrm{C}(\mathrm{gr}) \rightarrow \mathrm{H}_{2}(\mathrm{~g})+\mathrm{C}(\mathrm{gr})$ is 0.248 at $T_{\mathrm{g}}=100 \mathrm{~K}$ and $T_{\mathrm{s}}=10 \mathrm{~K}$, and the $\mathrm{H}_{2}$ formation occurs in a direct collision between $\mathrm{H}(\mathrm{g})$ and $\mathrm{H}(\mathrm{ad})$ on a subpicosecond scale. It can be compared with the probability of 0.534 at different thermal conditions for the same system $\left(T_{\mathrm{g}}\right.$ $\left.=1500 \mathrm{~K} . T_{\mathrm{s}}=300 \mathrm{~K}\right){ }^{16}$ The ensemble-averaged reaction time is 0.198 ps. Thus the recombination takes place efficiently at such an extreme thermal condition which mimicks the interstellar medium. But. it is interesting to note that this probability is significantly smaller than that for the hydrogen chemisorbed surface. That is, Farebrother $e t a l^{18}$ showed that at the gas temperature $100 \mathrm{~K}$ for which the most probable energy is $0.013 \mathrm{eV}$, the recombination is more efficient for the hydrogen chemisorbed surface. Somewhat larger probabilities 0.4-0.6 are found at the collision energy $0.1 \mathrm{eV}$ in the calculation by Panneix and Brechignac. ${ }^{15}$ who have used $D_{C \mathrm{H}}=0.665 \mathrm{eV}$, while Farebrother et al. used $D_{C \mathrm{H}}$ $=1.58 \mathrm{eV} \cdot{ }^{18}$ On the other hand, the probability based on $D_{C \mathrm{H}}$ $=0.57 \mathrm{eV}$, the calculations of Jeloaica and Sidis ${ }^{17}$ represent- 


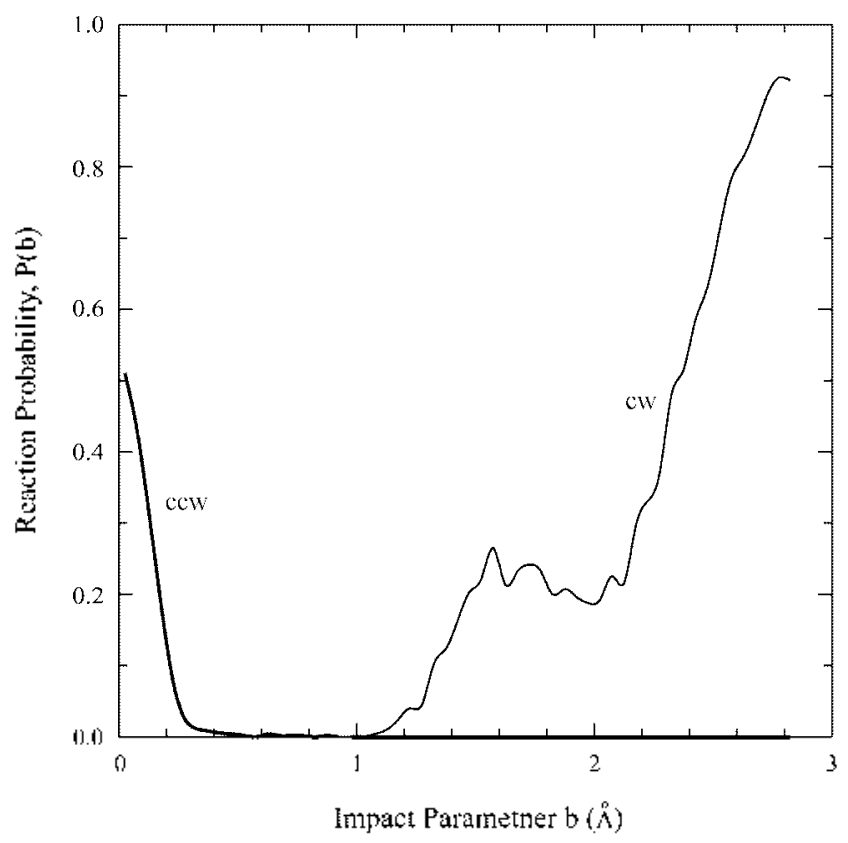

Figure 2. Dependence of the reaction probability $P(b)$ on the inpact paranneter $b$. The thick solid line is for the cw orientation and the thin solid line for ccw.

ing relatively weak chemisorption sites. is 0.503 at $T_{\mathrm{g}}=100$ $\mathrm{K}$ and $T_{\mathrm{s}}=10 \mathrm{~K} . ?$

In Figure 2. we show the dependence of $\mathrm{H}_{2}$ formation on the impact parameter in the range from $b=0$ to $b_{\max }=2.84$ $\AA$. (We will discuss the two curves denoted by "cw" and "ccw" in the next section.) Except near $0.2<b<1.2 \AA$ collisions. the opacity function takes a significant value. in particular in the collisions where $b>2.4 \dot{A}$. Nine carbon sites are configured in the range between this distance and $2 d \mathrm{cc}=2.842 \AA$. This result indicates that $\mathrm{H}(\mathrm{g})$ approaching close proximity to the surface with an impact parameter falling in this range tends to move toward $\mathrm{H}(\mathrm{ad})$ on $\mathrm{C}_{(0)}$. At short range. the surface atoms produce a repulsive wall and steer $\mathrm{H}(\mathrm{g})$ in the direction of $\mathrm{H}(\mathrm{ad})$ for $\mathrm{H}_{2}$ formation. We note that the total reaction cross section defined as $\sigma=$ $2 \pi \int P(b) b d b$ is as large as $9.22 \AA^{2}$. Sha and Jackson, using quantum scattering methods and the energy of the physisorbed $\mathrm{H}$ of $0.036 \mathrm{eV}$. reported cross section to be about $8 \mathrm{~A}^{2}$ around $E_{i}=0.1 \mathrm{eV}$ for the physisorbed $\mathrm{H}$ case. ${ }^{21}$

B. Reactive Trajectories. In Figures $3 a$ and $3 b$ we show two trajectories. each representing the representative trajectory producing the same direction of orientation of $\mathrm{H}_{2}$ when it begins the upward journey from the surface. To study this orientation problem. we first note that in both figures. the trajectories at the instant of the initial impact (i.e.. the first minimum of the $\mathrm{H}(\mathrm{g})$-surface distance) are below the $H(\mathrm{ad})$-surface distance. When $H(\mathrm{~g})$ approaches close range to the surface. its interaction with $\mathrm{H}(\mathrm{ad})$ weakens the H(ad)-surface bond. The upward motion of $H(\mathrm{ad})$ when $\mathrm{H}(\mathrm{g})$ approaches the first $\mathrm{H}(\mathrm{g})$-surface minimum represents this situation and the motion results in the $\mathrm{H}(\mathrm{ad})$-surface distance being increased by nearly $1 \mathrm{~A}$. As shown in Figure 3a. after reaching the first minimum $\mathrm{H}(\mathrm{g})$ begins to attract

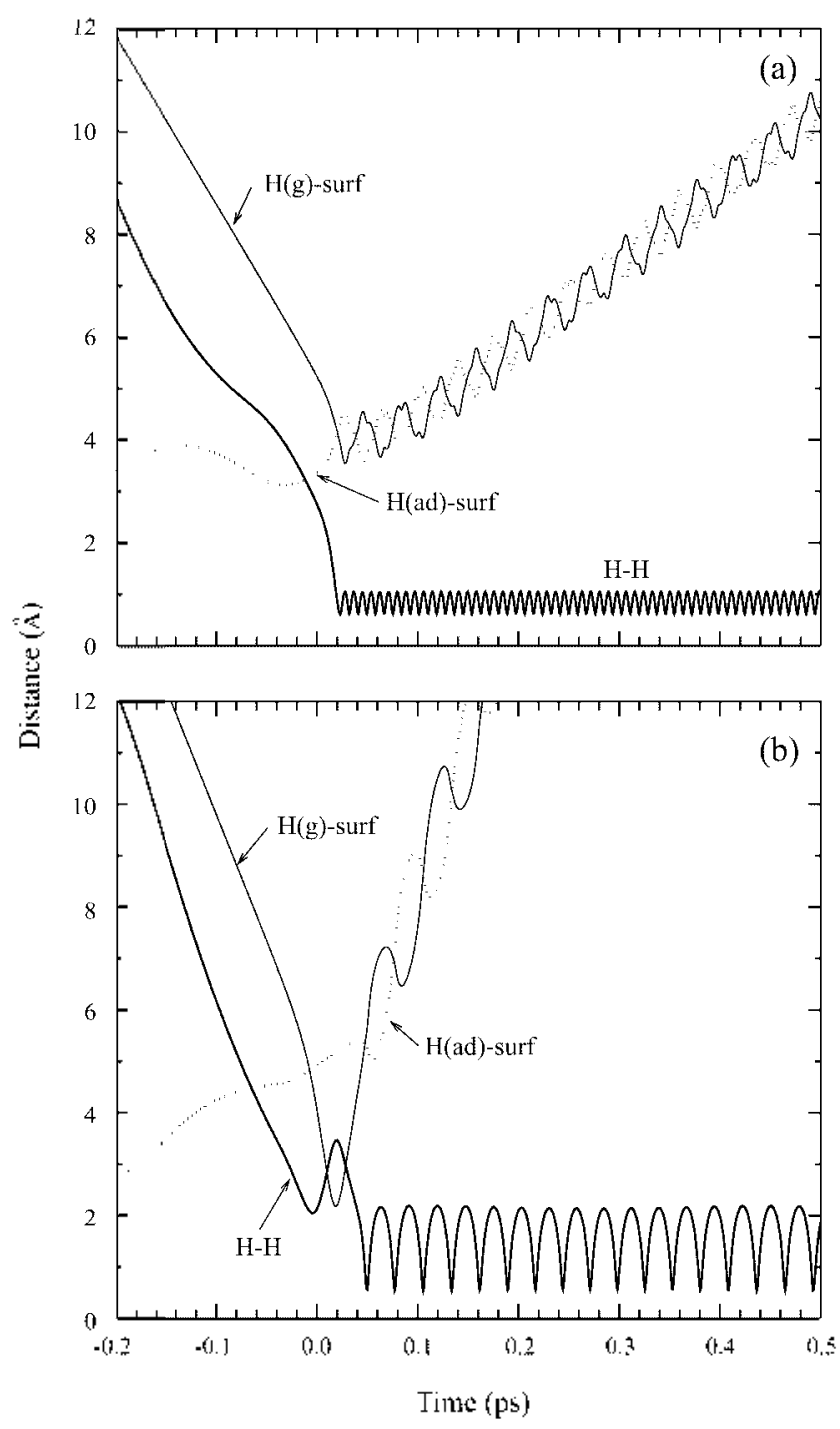

Figure 3. Time evolution of the H(g)-surface, $\mathrm{H}(\mathrm{ad})$-surface, and $\mathrm{H}(\mathrm{g})-\mathrm{H}(\mathrm{ad})$ distances for (a) the co orientation and (b) the ccw orientation.

the outgoing $\mathrm{H}(\mathrm{ad})$ to form $\mathrm{H}(\mathrm{g})-\mathrm{H}(\mathrm{ad})$. i.e... $\mathrm{H}_{2}$. The wellorganized oscillation of the $\mathrm{H}-\mathrm{H}$ distance beyond the first maximum confirms the formation of a stable $\mathrm{H}_{2}$ molecule. It is important to notice that the first minimum of the H(g)surface distance and the first maximum of the $\mathrm{H}(\mathrm{ad})$-surface distance occur at nearly the same instance. thus indicating that $\mathrm{H}_{2}$ forms with nearly end-on (or vertical) geometry with $\mathrm{H}(\mathrm{g})$ at the bottom. In Figure $3 \mathrm{~b} . \mathrm{H}(\mathrm{g})$ rebounding from the surface after the initial impact affects the adatom to form $\mathrm{H}_{2}$. In this case. unlike the previous case shown in Figure 3a. $\mathrm{H}(\mathrm{ad})$ is now closer to the surface at the instant of the $\mathrm{H}_{2}$ formation. For some trajectories. the $\mathrm{H}(\mathrm{g})$-surface and $\mathrm{H}(\mathrm{ad})$-surface distances are not significantly different from each other, but the $H(\mathrm{ad})$ end is still closer to the surface. We now discuss this difference between the two representative trajectories.

For the representative trajectory shown in Figure 3a. H(g) at the second minimum is as close to the surface as that at the ninimum (the first impact) at which $\mathrm{H}_{2}$ is formed. But 
thereafter the minima of the $\mathrm{H}(\mathrm{g})$-surface distance diverge. In a nonzero- $b$ collision. $\mathrm{H}(\mathrm{g})$ of the rotating $\mathrm{H}_{2}$ immediately after the $\mathrm{H}_{2}$ formation is attracted toward the surface site which has just released $\mathrm{H}(\mathrm{ad})$. As the nascent molecule begins its journey away from the surface. this attraction causes the $\mathrm{H}(\mathrm{g})$ end to move toward the axis normal to the

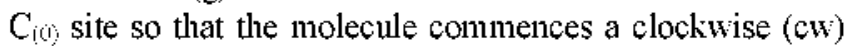
rotation. In particular. since the forces of the subsequent impacts (second in the representative case) exert a large torque. the receding molecule is hurled into a high rotational state with the cartwheeling-type rotation. A similar result has been observed in the scattering of $\mathrm{N}_{2}$ on $\operatorname{Ag}(111) .7$ Nearly $89 \%$ of the reactive trajectories undergo this type of rotation. In this case the reaction takes place on a subpicosecond scale when $\mathrm{H}(\mathrm{g})$ reaches the first turning point. so the incident angle determines the angle at which $\mathrm{H}_{2}$ forms.

In Figure $3 \mathrm{~b}$. on the other hand. the second minimum of the $\mathrm{H}(\mathrm{g})$ surface distance is now much farther away from the surface than the first minimum. It is more than $2 \mathrm{~A}$ farther. As shown in Figure 3b. the dynamics of this representative case is very different from that of Figure 3a. In particular, the incident atom approaches the surface much closer than in the case shown in Figure 3a and then rebounds to a larger distance before it is attracted back by $\mathrm{H}(\mathrm{ad})$ to form $\mathrm{H}_{2}$. In this case. when the $\mathrm{H}(\mathrm{ad})$-surface bond breaks and $\mathrm{H}_{2}$ forms. the $\mathrm{H}(\mathrm{ad})$ end of $\mathrm{H}_{2}$ tends to move away from the surface site. thus causing the nascent molecule to begin a counterclockwise (ccw) motion. The product molecule for the rest of reactive trajectories $(11 \%)$ undergoes this type of orientation. We now note that as $\mathrm{H}(\mathrm{g})$ turns around the corner at the closest approach and bounces back to the first maximum. a significant amount of acceleration of the trajectory occurs. This acceleration produces strong vibrational excitation as seen by a large amplitude motion with a large vibrational period of the $\mathrm{H}-\mathrm{H}$ distance beyrond the first maximum of the trajectory (compare the $\mathrm{H}-\mathrm{H}$ distance in Figure $3 \mathrm{~b}$ with that in 3a).

Now we return to Figure 2 to discuss the $\mathrm{cw}$ and $\mathrm{ccw}$ curves. The hydrogen molecule produced in a large- $b$ collision has a strong tendency to rotate clockwise. Where $b$ $>2.4 \mathrm{~A}$ the probability of forming such molecules is significantly large. This range is $\sqrt{3} d \mathrm{cc}<b<2 d c \mathrm{c}$. corresponding to the region near $\mathrm{H}-\mathrm{C}_{i,}, i=2,3,4,6,7,8,10,11$ and 12 . However. in $\mathrm{H}(\mathrm{g})-\mathrm{H}(\mathrm{ad})$ interactions where the impact parameter is smaller than $1.0 \mathrm{~A}$. the probability of forming cw molecules diminishes. On the other hand. the ccw probability takes maximum values near $b=0$. and decreases to zero in the region where the impact parameter is larger than $0.4 \AA$. In Figure 2 . the ccw and cw curves show their maximum near $b=0$ and $2.8 \AA$. respectively corresponding approximately to the regions near $\mathrm{H}(\mathrm{ad})-\mathrm{C}_{(\mathrm{cis})}$ and near $\mathrm{H}-\mathrm{C}_{(\mathrm{i})}$. $i=2,3,4,6,7,8,10,11$ and 12 , respectively. This calculated rotational orientation arises from corrugation in the gassurface interaction potential.

The dynamics of $\mathrm{H}(\mathrm{g})$ approaching the surface closer than the H(ad)-surface separation implies that the incident angle.

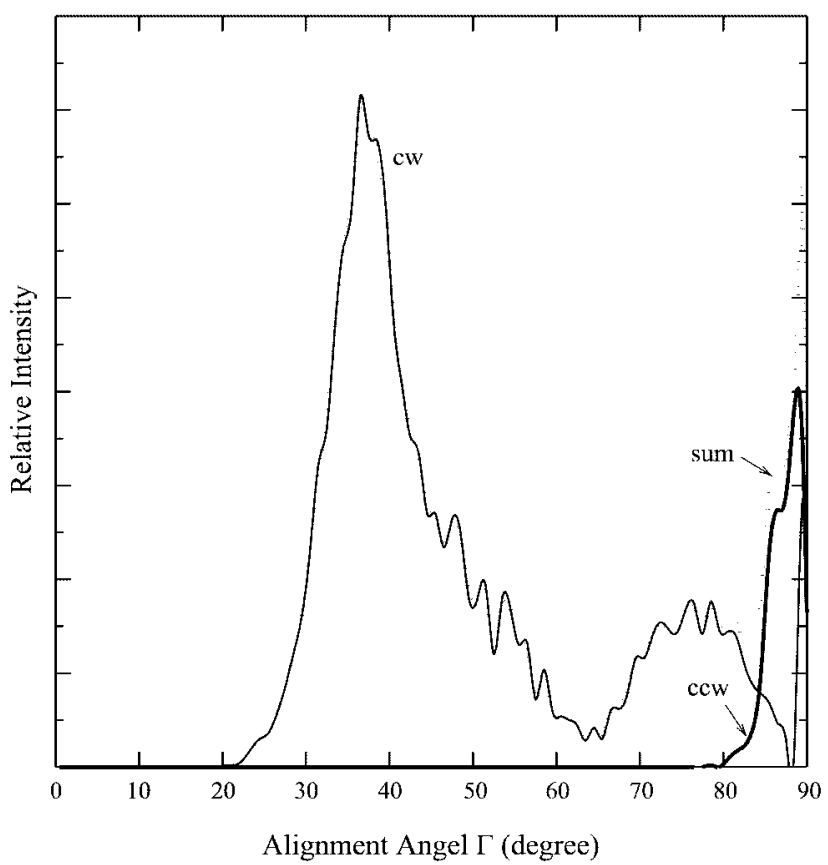

Figure 4. Relative intensity distribution of the alignment angle $\Gamma$ for the molecules undergoing $\mathrm{cw}$ and $\mathrm{ccw}$ orientations. The dotted lines is the sum of cw and ccw components.

$\theta_{\text {ntc }}$ defined in Figure 1. is larger than $90^{\circ}$. In a direct collision. which takes place on the subpicosecond scale. the value of this angle at the instant of the $\mathrm{H}_{2}$ formation is strongly correlated with the alignment of the $\mathrm{H}_{2}$ rotational axis. The angle $\Gamma$ between the axis of rotation of $\mathrm{H}_{2}$ and the surface normal at the instant of $\mathrm{H}_{2}$ formation can be determined from this angle. and its distribution provides the information on the rotational alignment of $\mathrm{H}_{2}$. In Figure 4. we plot the distribution of $\Gamma$ for all reactive trajectories. The $\mathrm{cw}$ case has the maximum distribution near $35^{\circ}$. That is. when $\mathrm{H}(\mathrm{g})$ is closer to the surface and binds with $\mathrm{H}(\mathrm{ad})$. the product molecule tends to leave the surface in an intermediate state between the cartwheel-like and helicopter-like rotations. It is interesting to compare this result with the cartwheeling-type rotation of $\mathrm{N}_{2}$. when it scatters from $\mathrm{Ag}(111)$ in nearly end-on geometry. ${ }^{-9} \mathrm{On}$ the other hand. for the majority of $\mathrm{H}_{2}$ leaving the surface with ccw rotation. the rotational axis is over $80^{\circ}$, in particular the maximum distribution occurs near $90^{\circ}$. indicating cartwheel-like rotations when $\mathrm{H}(\mathrm{ad})$ is closer to the surface and binds with $\mathrm{H}(\mathrm{g})$ in nearly head-on geometry. The molecule. which starts out with such head-on geometry. is not particularly efficient in carrying a large amount of rotational energy. As shown in Figure 4 , we find no product molecules with the rotational axis perpendicular to the surface.

C. Vibrational and Rotational Energies. The difference between the $\mathrm{H}-\mathrm{H}$ and $\mathrm{H}$-surface interaction energy is 4.751 $\mathrm{eV}$. so the energy available to the product state is $4.751+E-$ $E_{C H}^{i}$. The vibrational motion is found to share most of the reaction exothermicity thus producing vibrationally highly excited $\mathrm{H}_{2}$ molecules. The ensemble-averaged vibrational energy of $\mathrm{H}_{2}$ is as large as $3.43 \mathrm{eV}$. 


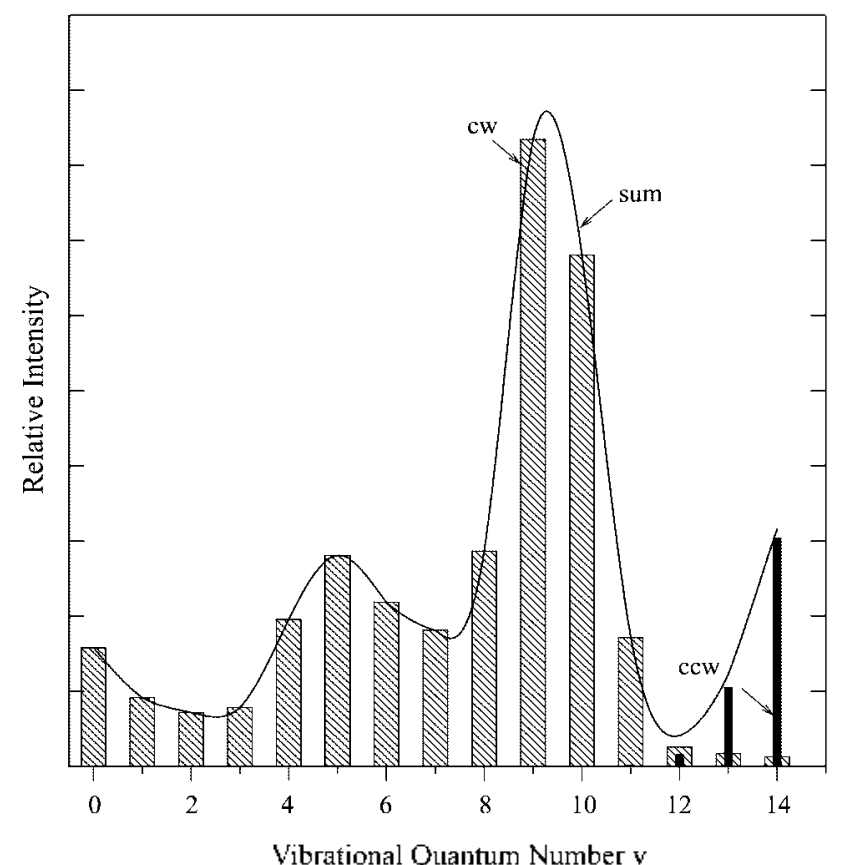

Figure 5. Relative intensity distribution of the vibrational population for the cw and ccw molecules. The solid line is the sum of $\mathrm{cw}$ and cew components.

The vibrational energy of $\mathrm{H}_{2}$ for the representative trajectory considered in Figure $3 \mathrm{a}$ is $0.750 \mathrm{eV}$. whereas that in Figure $3 \mathrm{~b}$ is $3.45 \mathrm{eV}$. Such a large difference can be expected from a qualitative comparison of the $\mathrm{H}-\mathrm{H}$ distance shown in the two figures. Here we have determined the energy deposited in the vibrational motion of $\mathrm{H}_{2}(\mathrm{~g})$ from the usual expression $E_{\text {vib }}=p^{2} / 2 m_{H_{2}}+D_{H H}\left[1-e^{i}{ }^{H} H \mathrm{H}^{-} \cdot \mathrm{HH}^{i}: \alpha_{H H}\right]^{2}$. sum of kinetic and potential energies. To mimic the quantum vibrational distribution. we use a binning procedure of assigning quantum number $v$ corresponding to $E_{\mathrm{v} i \mathrm{~b}}$ through the relation $v=\operatorname{int}\left[E_{\text {vib }} / E(v)\right]$. corresponding to the values of Kolos et $a l^{30}$ As shown in Figure 5, the intensity of vibrational population distribution for hyydrogen molecules produced in the reaction is trimodal (see the curve for the sum). which is an overlap of the distributions of $\mathrm{cw}$ and $\mathrm{ccw}$ rotating molecules. The population distribution is the largest for $v=9$. but the intensity is still significant event near the dissociation threshold. On the other hand. it is interesting to note that the vibrational excitation for the chemisorbed case $^{16}$ shows the population maximum appearing at $v=3$. In general. for both $\mathrm{cw}$ and $\mathrm{ccw}$ cases vibrational energies are highly excited and the resulting population deviates seriously from the Boltzmann distribution. In particular. highly' vibrationally excited states are dominated by $\mathrm{H}_{2}$ rotating $\mathrm{ccw}$. As noted above in Figure 3b. a significant amount of acceleration of the trajectory occurs after turning the closest approach, resulting in such high vibrational excitation.

The distributions of rotational population are shown in Figure 6 . where the rotational quantum number $J$ corresponding to the calculated energy $E_{\text {rot }}$ is determined using the same binning procedure introduced above: $J=\operatorname{int}\left[E_{\text {rot }} /\right.$ $E(J)]$ with $E_{\mathrm{rOt}}=L^{2 / 2} \mu_{\mathrm{HH} / \mathrm{HH}}{ }^{2}$ and $E(J)=J(J+1) \hbar^{2} / 2 I_{\mathrm{HH}}$.

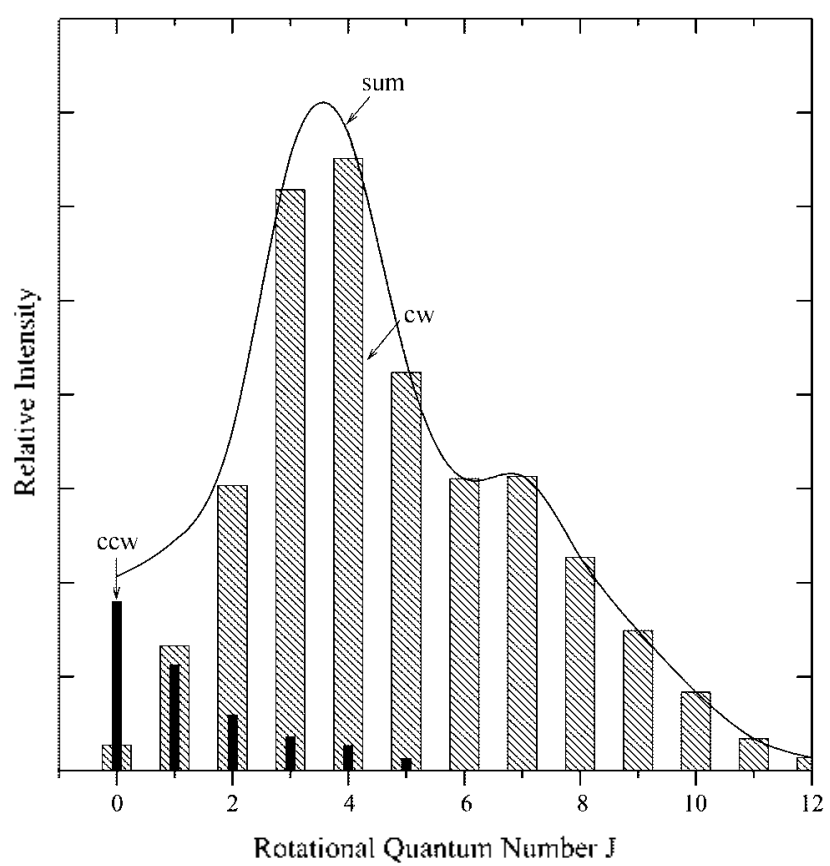

Figure 6. Relative intensity distribution of the rotational population for the $\mathrm{cw}$ and cew molecules. The solid line is the sum of $\mathrm{cw}$ and cow components

where $L$ is the angular momentum $\mu_{\mathrm{HH}}(\mathrm{z} \dot{\rho}-\rho \dot{z})$. The $\mathrm{cw}$ trajectory receives a torque from the steep side of the $\mathrm{H}(\mathrm{g})$ surface interaction as the $\mathrm{H}(\mathrm{g})$ end rapidly goes around the turning point (see Figure 3a). As a result. a closer approach of $\mathrm{H}(\mathrm{g})$ to the surface at the second impact after the first full rotation results in strong rotational excitation. These $\mathrm{cw}$ molecules begin their orientation and alignment when the two atoms combine in nearly end-on geometry and the highest cw population occurs at $J=4$. However the distribution of $\mathrm{ccw}$ rotational population is concentrated at lower rotational states. Since low rotational excitation leaves more energy for vibration. a ccw rotating molecule usually has a larger amount of vibrational energy compared with a $\mathrm{cw}$ molecule. The ensemble-averaged vibrational energy is 4.69 $\mathrm{eV}$ for the ccw molecules. but the corresponding energy is only $3.27 \mathrm{eV}$ for the cw case.

\section{Concluding Comments}

We have studied the recombination of gaseous hydrogen atoms with weakly adsorbed hydrogen atoms on a graphite surface at the thermal conditions mimicking the interstellar medium. The recombination reaction is very efficient and $\mathrm{H}_{2}$ formation occurs on a subpicosecond time scale. Product molecules are strongly vibrationally excited and their population deviates seriously from the Boltzmann distribution.

The rotational motion of $\mathrm{H}_{2}$ can be oriented either clockwise or counter-clockwise. We have determined the orientation by following the trajectories at the very early stage of recombination. Clockwise orientation results for the molecule forming from near end-on geometry on the surface. The molecules rotating clockwise are in low-lying vibrational 
levels. However the molecules rotating counter-clockwise have the rotational alignment closely parallel to the surface with weak rotational excitation. The molecules with rotational motion oriented counter-clockwise are highly vibrationally excited. The number of molecules oriented clockwise is significantly larger than that of molecules oriented counterclockwise.

Acknowledgment Computational time was supported by a NSF Advanced Computing Resources grant at the San Diego Supercomputing Center and by the 3rd Supercomputing Application Support Program of the KISTI (Korea Institute of Science and Technology Information). J. R. gratefully acknowledges the financial support from the Chomuam National University in his sabbatical year of 2001.

\section{References}

1. (a) Rettner. C. T. Phys. Rev: Lett. 1992. 69.383. (b) Rettner. C. T.: Lee, I. J. Chem. Phrs 1994, 101, 10185.

2. Schermann, C.: Pichol. F.: Landau. M.: Cadez. I.: Hall, R. I. J. Chem. Phes 1994. 101.8152.

3. Jachimowski. T. A.: Weinberg. W. H. J. Chem. Plys. 1994. 101. 10997.

4. Shin. H. K. Chem. Phs. Lett. 1995. 24. 235.

5. Kratzer P. J. Chent. Plns. 1997, 106.6752.

6. Buntio. S. A. J. Chem. Phus 1998, I08. 1601

7. Lim, S. H. Ree, J.: Kim, Y. H. Bull Korean Chem. Soc 1999, 20. 1136

8. Ghio. E: Mattera. L.: Salvo. C.: Tommasini. F.: Valbusa. E. J. Chem. Phys. 1980. 73, 556 .
9. Hollenbach, D. H.: Salpeter. E. E. J. Chem. Phys. 1970, $53,79$.

10. Hunter. W. A.: Watson. W. D. Astrophy. J. 1978. 226. 477

11. Duley. W. D.: Williams. D. A. Interstellar Chemistry: Academic Press: Londont. 1984.

12. Williams. D. A. in Bailey. M. E.: Williams. D. A.. Eds.: Dust in the Lhinerse, Cambridge Univ. Press: Cambridge, 1988; $\mathrm{p} 391$

13. (a) Fitzpatric. E. L.: Masa. D. Astrophys. J. Stippl. Ser 1990.72 163. (b) Papoular, R.: Conrad. J.: Guillois. O.: Nenner. I.: Reynaud. C.: Rouzard. J.-N. Astom. Astrophys. 1996. 315. 222.

14. Guillois. O.: Ledoux. G.: Nenner. I.: Papoular. R.: Reynaud. C. Faraday Discuss. 1998. 109. 335.

15. Parneix, P; Bréchignac. Ph. Astonn Astrophns 1998, 334, 363.

16. Kim. Y. H. Ree. J.: Shin. H. K. Chem. Phn Lett 1999, 31t. 1.

17. Jeloaica, L.: Sidis. V. Chent. Phys Lett 1999, $300,157$.

18. Farebrother. A. J.: Mejer. A. T. H. M.: Clary. D. C.: Fisher. A. T. Chem. Phus. Lett. 2000. 319. 303.

19. Jackson. B.: Lemoine. D. J. Chem. Plhs 2001. 114. 474.

20. Ree, J.: Kim, Y. H.: Shin, H. K. Chem. Phts Lett. 2002, 353.368.

21. Sha, X; Jackson. B.: Lemoine. D. J. Chent Phis. 2002, 116. 7158 .

22. Adelmant. S. A. J. Chem. Phws. 1979.71. 4471.

23. Ree. J.: Shin. H. K. J. Cham. Phys. 1999. H1. 10261.

24. Huber. K. P.: Herzberg. G. Constants of Diatomic Holecules. Van Nostrand Reinhold: New York, 1979.

25. Shin. H. K. J. Chent Phws. 1978. 68, 335.

26. Kim. Y. H.; Ree. J.: Shin. H. K. J. Chent Ph.s. 1998. 108, 9821.

27. American Institute of Plnsics Handbook. 3rd ed.: Gray. D. E.. Ed: McGraw-Hill: New York. 1972: pp 4-115.

28. Ree. J.: Kim. Y. H.: Shin. H. K. J. Chem. Phys. 1996. 104. 742.

29. Kummel. A. C.: Sitz, G. O.: Zare. R. N.: Tully. J. C. J. Chent. Pho 1988, $89,6947$.

30. Kolos. W: Szalewicz. K.: Monkhorst. H. J. J. Chent. Phs 1986. 84.3278 . 